\title{
Design Considerations for Residents with Impeded Cognitive Functioning: Conversations with People with Schizophrenia
}

\author{
Elizabeth Karol * and Dianne Smith
}

check for updates

Citation: Karol, E.; Smith, D. Design Considerations for Residents with Impeded Cognitive Functioning: Conversations with People with Schizophrenia. Sustainability 2021, 13, 7733. https://doi.org/10.3390/ su13147733

Academic Editor: Rossano Albatici

Received: 18 June 2021

Accepted: 7 July 2021

Published: 11 July 2021

Publisher's Note: MDPI stays neutral with regard to jurisdictional claims in published maps and institutional affiliations.

Copyright: (c) 2021 by the authors. Licensee MDPI, Basel, Switzerland. This article is an open access article distributed under the terms and conditions of the Creative Commons Attribution (CC BY) license (https:/ / creativecommons.org/licenses/by/ $4.0 /)$.
DEKS Design Research Consultancy, Curtin University, Bentley 6102, Australia; Dianne.Smith@curtin.edu.au * Correspondence: elizabeth@deksdesignresearch.com.au

\begin{abstract}
Insights into the relationship between people with impeded cognitive functioning and the built environment have arisen over the last few decades. This research builds on underlying research that looked at how the home environment could help people feel supported so they could better cope with everyday living and thus achieve an enhanced QoL. Such support goes beyond functional design related to activities of daily living and encompasses opportunities for comprehension, management, finding meaningfulness in, and providing emotional affordance in the home. This article reports on conversational interviews with people with schizophrenia discussing their experiences of home environments. Their responses provide a basis on which to generate a more nuanced understanding of the impact of accommodation on people with schizophrenia, challenge considerations previously identified and highlight the need for further research. Considerations include the importance of the home's proximity to places for potential relaxation, the need for security of housing tenure rather than short-term crisis housing, awareness of the support required for occupants to maintain outdoor areas and enabling occupants to pursue personal interests in the home. The findings will inform the development of accommodation design considerations that focus on improving quality of life for people with schizophrenia. In this way, the findings will contribute to enabling this neurodiverse group to remain independent, empowered and functioning members of the community-leading to greater social sustainability.
\end{abstract}

Keywords: design; architecture; accommodation; schizophrenia; neurodiverse

\section{Introduction}

This paper reports on a study where people with schizophrenia were invited to talk about their experiences of homes they had lived in. The aim was to identify aspects of the environment that influence their sense of well-being in their daily lives. This provided an insider perspective of the particular aspects of a person's home that could be addressed in future design work to improve residents' quality of life.

Before relating the findings arising from these conversations, the context for this study is described. This includes the researchers' practice for responsive design of accommodation for people with impeded cognitive functioning, current influences in the field of design for disability in Australia, and schizophrenia as an example of impeded cognitive functioning. The design of the study is then outlined.

\section{Background of Study}

People with impeded cognitive functioning (ICF) may have an on-going impairment in comprehension, reasoning, adaptive functioning, judgement, learning or memory. A generic term for this variety of conditions is neurodiversity. People displaying these conditions may unnecessarily suffer in their home environments, directly or indirectly exacerbating their conditions, because they are unable to interpret environmental cues for what is happening, what may be expected and how to act.

For these people it is particularly important that their everyday environment is orderly, easy to understand and supportive of their psycho-social needs, as well as providing 
opportunities for them to enhance their quality of life (QoL) and to develop a sense of coherence (SoC). The authors propose that truly responsive accommodation design (RAD) practice can help to both improve QoL and empower these occupants. A fundamental tenet of this approach is to harness the residents' subjective experiences of home.

\subsection{Design Proposition: RAD-ICF Approach}

Overall a designer needs to focus on design solutions that address multiple design challenges simultaneously and thus achieve an outcome that best suits the resident and their particular context. An informed awareness of the condition (and what the person may be experiencing) is integral to a RAD-ICF design process. It is the experiences of people living in their homes that are the focus of the current study, to ensure that we as researcher-designers are not only informed about the particular condition of ICF but that we also have insights into how a resident's life is impacted from his or her perspective.

All parties engaged in using RAD-ICF are encouraged to actively support accommodation that is design-driven rather than purely efficiency-driven, involve users in the design process, commit to monitoring the performance of the completed building and imbed knowledge gained into future design projects across the sector. This process has evolved through ongoing research developed by the authors over the last decade. The preliminary work has been reported in [1-3]. We recommend that those engaging in RAD-ICF need to be invested in three critical approaches to the design of housing:

1. Firstly, being informed about ICF which may include 'an ongoing impairment in comprehension, reason, adaptive functioning, judgement, learning or memory' [4] (p. 136) and recognizing that a person with ICF may exhibit particular symptoms related to their condition in relationship with their environment;

2. Secondly, recognizing the importance of a sense-of-coherence (https: / / positivepsychology com/sense-of-coherence-scale/ accessed on 27 June 2018) in the design of a home where comprehensibility (the extent a person perceives stimuli as being understandable in a rational way, relying on an ability to see things coherently in a structured way with contextual referencing), manageability (the degree a person feels there are external and internal resources to help them manage incoming stimuli and deal with challenges), meaningfulness (the extent a person feels that their lives have some kind of emotional meaning which becomes relevant when facing a problem or challenge) and emotional affordance (the potential of an environment to foster an integrated emotional response by supporting a person's social-psychological needs and providing opportunities for aspects of a sense of self, sense of delight and a sense of control to arise) are all addressed; and

3. Thirdly, applying the available research in the form of design considerations [1] to initiate design strategies which enhance these residents' QoL whilst mitigating potential detrimental impacts within the home environment.

\subsection{The Design-For-Disability Context}

In Australia there have been two significant advancements over the past two decades in the area of accommodation for people with disabilities - the NDIS (National Disability Insurance Scheme 2013) and the Specialist Disability Accommodation (SDA) guidelines. These programs have broadened the discussion about design for people with a range of abilities. The schemes aim to provide funding to people so that they can have more control over both their care programs and their accommodation. The SDA provides guidelines for housing and a means to evaluate proposals for accommodation design 'specially designed or modified to suit the needs of people who have an 'extreme functional impairment' or 'very high support needs' [5] (p. 1). The residents of SDA housing include people with cognitive impairment connected to brain injury, spinal injury, stroke, chronic or degenerative disease and mental illness [6]. This shift in the context for supportive accommodation raised questions for architects and designers, as well as housing providers, regarding the form, quality and impact of existing and future housing options making it timely to re-evaluate current practices. 
Our research, which included a review of others' research [3] and design practice, focuses on solutions for those with impeded cognitive functioning. The majority of the research available at that time strove to identify the impact of the built environment, particularly homes, on people with schizophrenia. As Boys [7] (p. 1) flagged, 'rethinking ability and architecture offers a powerful tool to design differently;' that is, not just for accessibility and inclusive design which traditionally has been seen in education and practice as 'dull, dry, and yet another regulatory pressure constraining designers.' Rather, she asks, '[c]an disability-and ability-help us think more explicitly about habitation, how we envisage a 'typical' user and what impacts buildings have on their many and varied occupants?'

\subsection{Schizophrenia}

To create places that could positively support residents with schizophrenia in a variety of dimensions of their life [1,2] requires some understanding of schizophrenia. Aya et al. [8] (p. 4) explain that 'Schizophrenia impacts on some of the highly developed functions of the human brain and has three main categories of symptoms: psychotic or "positive" symptoms (aggressiveness, agitation, delusions, and hallucinations); deficit or "negative" symptoms (poverty in speech and major disturbances in social relations, total lack of motivation, inability to feel pleasure, and apathy); cognitive dysfunction symptoms (impaired attention, learning, and memory). The psychotic symptoms have an episodic pattern, but the negative and cognitive symptoms are more persistent and chronic.'

Those three categories of symptoms that could be directly or indirectly influenced by the environment were explored to ascertain how design solutions could potentially have a positive impact on well-being. For example, it is established that for a person with schizophrenia, any of the senses, including the auditory sense, can trigger hallucinations. Although noise does not cause mental health issues, it is reported [9] that noise is understood to 'accelerate and intensify the development of latent mental disorders' and may influence a number of 'adverse effects: anxiety, stress, nervousness, nausea, headache, emotional instability, argumentativeness, sexual impotence, changes in mood, increase in social conflicts, neurosis, hysteria, and psychosis' (p. 291). Thus accommodation design should enable a person to control noise levels, whether the source is external or internal, to avoid reactions such as agitation and/or anxiety [10].

\section{The Study}

The current study (reported below) discusses aspects of the environment that people with schizophrenia identify as being important to them. It is envisaged that such a usersensitive approach that focuses on the resident's relationship with the environment will support social and environmental sustainability and will better support a resident's ability not only to cope but to feel their well-being is enhanced.

\subsection{Intention}

The core objective was to identify how people who have been diagnosed with schizophrenia experience their residential environments. Emphasis was given to the impact of the physical setting on how well people felt supported in undertaking their daily lives and having a sense of well-being. The anticipated immediate outcome is a list of environmental aspects of homes that exert influence on people with schizophrenia and the associated impacts of each aspect. It is anticipated that in the long-term the findings will inform designers, researchers and accommodation providers about designing accommodation for people with impeded cognitive functioning.

\subsection{Methodology}

Theoretical studies drawn from such fields as environmental psychology, sociology and design research show that particular design features of an environment can positively support people with ICF [3]. Currently, typical design practitioners interpret 'support' as 
integrating mandatory prescriptive regulations and voluntary codes which focus on making accommodation easy to enter, easy to move in and around and easily and cost-effectively adapted to changing physical needs. Such 'support' addresses functional aspects of ADL. However, researchers [1] also identify the importance of abstract concepts to support well-being such as opportunities for self-determination, spatial diversity and experiencing spatial delight.

To help identify abstract concepts that are meaningful it is imperative the perspectives of those with ICF are heard [11]. Therefore, a qualitative research approach involving a diary and semi-structured interviews with people with ICF was adopted. Ethics approval to carry out these interviews was obtained from Curtin University.

\subsubsection{Participants}

All study participants have some difficulties with their cognitive functioning but were able to articulate their perceptions of their home environment. This contributed to our overall reflection on the essential environmental inclusions (architectural and interior). As this study is a qualitative study that is seeking in-depth information regarding experiences, the sample space is small. In keeping with this investigative mode a sample of 12 participants was initially proposed to ensure that the data would be saturated $[12,13]$. The logistics of enlisting participants, even via service providers, was not straightforward. Service providers are stretched to meet the needs of their clients and have little time to facilitate research work. Further, those with schizophrenia are frequently anxious about anything 'different' in their lives and may struggle to put themselves forward to participate in research. The final number of participants was ten face to face and one online interview. There were five female participants and six male participants varying in age from 30 to 66 .

A representative subsection of this population was not sought nor a particular response rate per environmental attribute. Rather the range of experiences and their impact for each participant was to be identified. In addition to building immediate knowledge, these comments were envisaged becoming aspects for future research. Therefore the eligibility criteria were that participants:

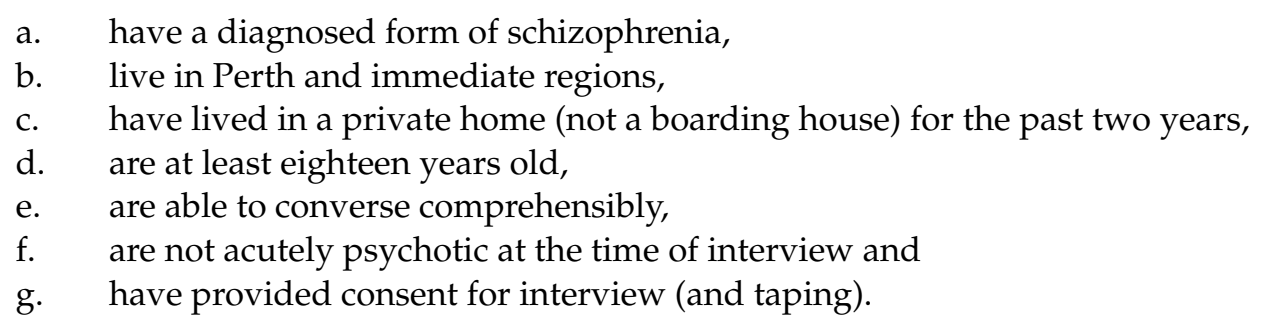

People were recruited via community groups or by snowball/word of mouth via contacts. Those interested in being involved were given a detailed information sheet and a consent form that they were asked to sign. Each was given a gift voucher following the interview.

\subsubsection{Methods}

Two methods were used: a diary and an interview. For the diary, a notebook was given to each participant one week prior to the face-to-face interview. During that week they were asked to make notes of anything that they noticed about their home, as well as their memories about previous homes, that impacted their QoL. This was to help prime the participant to focus on the built environment. They brought the diary to the interview session and the interviewer subsequently typed up the contents of the diary. If requested, the diaries were returned to the participant.

Both researchers were present at each interview. The conversational interview, held in the participant's home, lasted between $30 \mathrm{~min}$ and one hour. As participants were high functioning, fatigue was not envisaged to be a major issue. Each interview was taped and transcribed verbatim. Field notes were also taken by one researcher. The transcripts and field notes were cross-checked to ensure accuracy. 
Following a general greeting, each face-to-face interview was formatted into three parts. Lead-in questions provided a non-threatening preliminary interaction to gain background data about the participant's accommodation history. These were followed by open-ended questions to identify their experiences of past homes and their impact. A specific probe was added if the participant had not described the impact of the physical aspects of the home/s on their well-being (such as spaces, the layout, the entry, the rooms, finishes, decoration, lighting, atmosphere and landscaping). Finally, the current home was discussed. Participants were probed regarding enjoyable aspects (things about the home that made their life better), aspects they would most like to change, what helped or stopped them doing what they liked, particular areas in the home that they felt made things difficult and their expectations for qualities of future homes, and were asked to comment on the impact of the physical aspects of their current home on their well-being.

\subsubsection{Process of Analysis}

In summary, each researcher read each transcript independently twice-firstly to identify concepts arising in it and secondly to code the concepts into one of three categories: design considerations, impact on the person and general issues explicitly associated with schizophrenia. The codes were then jointly compared and refined.

The participants' verbal accounts of their experiences were analyzed to identify the general themes and the key concepts arising therein. The first reading was intentionally broad so that the voices of the participants were able to be captured, thereby not restraining concepts to the researchers' predetermined intentions or biases. This was in the spirit of research in grounded theory [14-16], where the researcher seeks to identify concepts which could provide new insights into a situation or phenomena.

These initial concepts were then coded and recorded in one or more of the three categories mentioned above: those relating to design features (or the more functional aspects), those relating to the impact on well-being (or the more intangible aspects) and those general issues connected with schizophrenia per se. A limited number of the key concepts were noted in more than one category when more than one interpretation of the concept was identified.

This process involved the researchers revisiting the coding following three blocks of interviews (\#1-5) (\#6-8) (\#9-11) to ensure that the codes arising from the data were sensitive to the nuances embedded in all conversations. This provided for in-depth reflections on the context of each of the comments and allowed their possible implications to be interrogated. These steps resulted in some codes being merged or recategorized for clarity. The final primary and secondary codes related to functional aspects of the home and the impact on the well-being of the person are shown in Section 3, Figure 1.

The final key concepts were also compared with an earlier framework created as part of the RAD-ICF design process [1] that sought to identify design principles associated with aspects of schizophrenia in order to provide an environment with a strong SoC. The initial RAD-ICF principles had been divided into four clusters relating to SoC (comprehension, manageability, meaningfulness and emotional affordance), and for each cluster a range of design provisions had been identified. New understandings drawn from the current analysis were used to reflect upon these provisions. In addition, the impact on well-being for people with schizophrenia had been identified from the literature and linked to each principle and associated provisions and served as a checklist to review the findings noted below. Thus, the current analysis will enable the further refinement and development of the framework used in the RAD-ICF design process. 


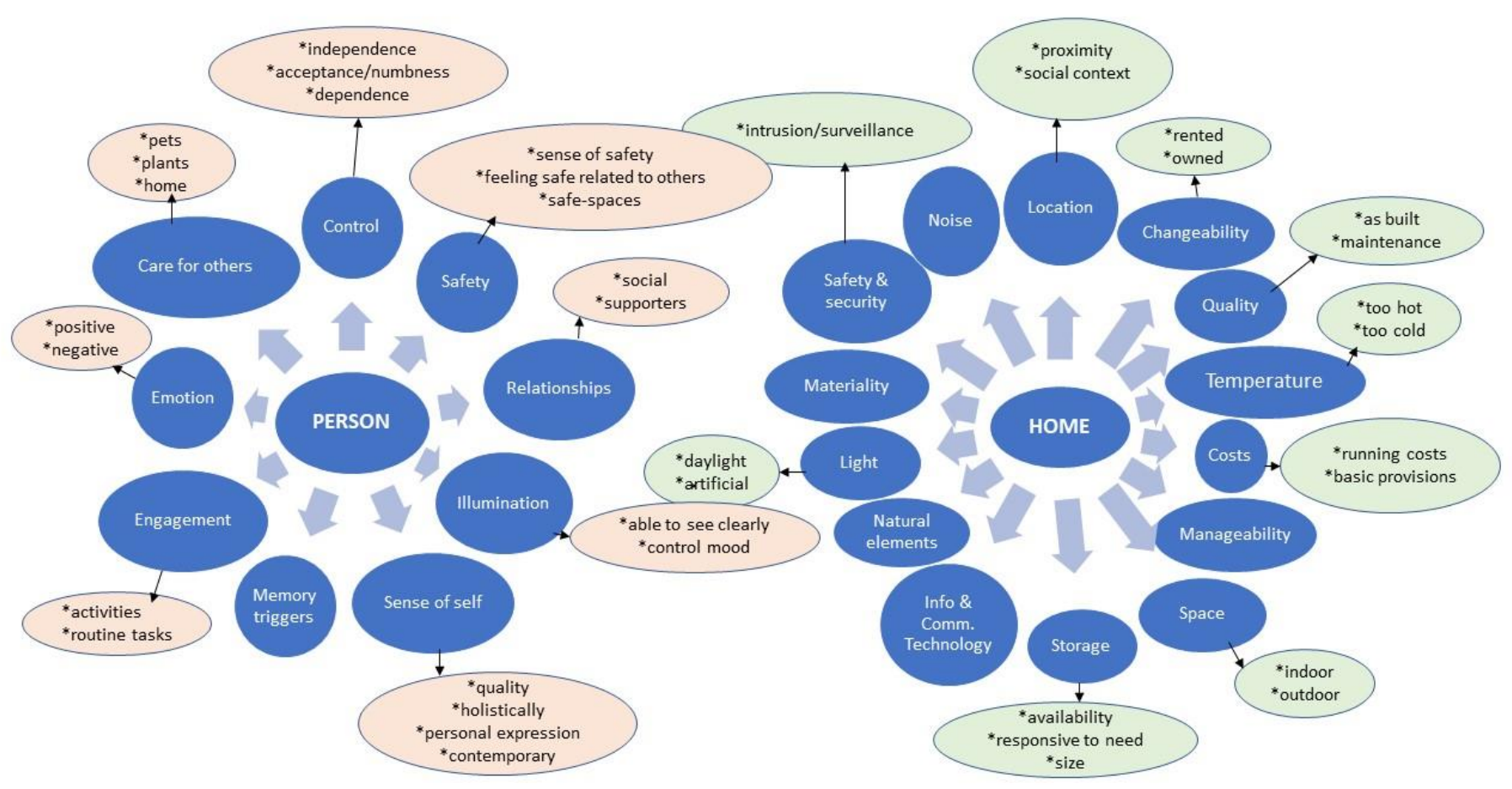

Figure 1. Summary of concepts raised related to person and home.

\section{Findings}

The premise for this study was that individuals with schizophrenia respond to the environment in similar ways to everyone else, yet these participants' experiences are filtered through or moderated by the particulars of their condition in association with their past experiences. Two core aspects are involved. Firstly, the participant's response involves personal characteristics such as the person's feelings, levels of competency and overall well-being. In the context of this study, how these personal characteristics may affect or be affected by a participants' relationship with the environment (that is, the home setting), also needs to be identified. Secondly, in contrast to such emotional aspects, the more physical aspects of the accommodation experience are implicated. These include aspects such as room size, access paths, lighting level and/or views. However, both these aspects work in combination to influence the QoL and sense of well-being, and, as a consequence, the amount of support needed for a resident with schizophrenia to live to their maximum potential. We sought to identify if these aspects were captured through the discussions of the participants' experiences.

Primary, secondary and tertiary levels of codes were identified. These codes were divided into those associated with the impact on home design (Table 1) and the impact on the person (Table 2). The right-hand column in each table indicates the strength of the response (low, medium or high) to each aspect of the participants' experiences. The experiences were mentioned in either positive or negative terms.

Figure 1 provides a summary of what participants discussed as affecting or being affected by the home environment. It can be seen in Figure 1 that there are two overlaps between the concepts related to the impact on home design and the impact on the person. The overlaps relate to the way a home design enables certain lighting conditions which a person may perceive as impacting positively or negatively on their well-being and how having control of physical aspects of home security impacts on feelings of safety in various situations. 
Table 1. Coding of concepts related to impact on home design.

\begin{tabular}{|c|c|c|c|}
\hline Primary & Secondary & Tertiary & Strength of Response \\
\hline \multirow{3}{*}{ Changeability } & \multirow{2}{*}{ Rented } & Permitted & low \\
\hline & & Feasible & low \\
\hline & Owned & Feasible & medium \\
\hline \multirow{2}{*}{ Costs } & Running costs & & high \\
\hline & Basic Provisions & & low \\
\hline Inform. \& comm. tech. & & & medium \\
\hline \multirow{2}{*}{ Light } & Daylight & & high \\
\hline & Artificial & & low \\
\hline \multirow{6}{*}{ Location } & \multirow{5}{*}{ Proximity } & To services & high \\
\hline & & To transport \& parking & medium \\
\hline & & To blue/green areas & low \\
\hline & & To noisy surrounds & low \\
\hline & & To ground level & low \\
\hline & \multirow[t]{2}{*}{ Social context } & & high \\
\hline Manageability & & & medium \\
\hline Materiality & & & medium \\
\hline Natural elements in immediate vicinity & & & medium \\
\hline Noise & & & low \\
\hline \multirow{3}{*}{ Quality } & As built & & low \\
\hline & \multirow{2}{*}{ Maintenance } & Ongoing & medium \\
\hline & & Renovation & low \\
\hline \multirow{2}{*}{ Safety \& security } & \multirow{2}{*}{ Intrusion/surveillance } & Physical & high \\
\hline & & Visual & low \\
\hline \multirow{4}{*}{ Space } & \multirow{2}{*}{ Indoor } & Physical size & high \\
\hline & & Useability of space & high \\
\hline & \multirow{2}{*}{ Outdoor } & Physical size & low \\
\hline & & Useability of space & medium \\
\hline \multirow{3}{*}{ Storage } & Availability & & medium \\
\hline & Size & & low \\
\hline & Responsive to need & & medium \\
\hline \multirow{2}{*}{ Temperature } & Too hot & & medium \\
\hline & Too cold & & low \\
\hline
\end{tabular}


Table 2. Coding of concepts related to impact on person.

\begin{tabular}{|c|c|c|c|}
\hline Primary & Secondary & Tertiary & Strength of Response \\
\hline \multirow{6}{*}{ Care for other } & \multirow{2}{*}{ Pets } & Supportive aspects & medium \\
\hline & & Inhibiting aspects & low \\
\hline & \multirow{2}{*}{ Plants } & Supportive aspects & low \\
\hline & & Inhibiting aspects & low \\
\hline & \multirow{2}{*}{ Home } & Supportive aspects & low \\
\hline & & Inhibiting aspects & low \\
\hline \multirow{3}{*}{ Control } & Independence & & medium \\
\hline & Dependence & & medium \\
\hline & Acceptance/numbness & & low \\
\hline \multirow{4}{*}{ Emotion } & \multirow{2}{*}{ Negative emotions } & Being overwhelmed & low \\
\hline & & General & medium \\
\hline & \multirow{2}{*}{ Positive emotions } & Affects well-being & medium \\
\hline & & General & low \\
\hline \multirow{3}{*}{ Engagement } & Routine tasks & & low \\
\hline & \multirow{2}{*}{ Activities } & For self-maintenance & medium \\
\hline & & For satisfaction & high \\
\hline \multirow{2}{*}{ Illumination } & Able to see things & & low \\
\hline & Controls mood & & low \\
\hline Memory triggers & & & medium \\
\hline \multirow{4}{*}{ Relationships } & \multirow{3}{*}{ Social } & Neighbours & medium \\
\hline & & Friends & medium \\
\hline & & Family & medium \\
\hline & Support workers & & low \\
\hline \multirow{4}{*}{ Safety } & \multirow{2}{*}{ Sense of safety } & Emotional safety & medium \\
\hline & & Medical safety & low \\
\hline & $\begin{array}{l}\text { Feeling safe/unsafe related to others } \\
\text { in household }\end{array}$ & & low \\
\hline & Safe-spaces & & low \\
\hline \multirow{4}{*}{ Sense of self/identity/self-worth } & Holistically & & medium \\
\hline & Quality related & & low \\
\hline & Contemporary feel & & low \\
\hline & Personal expression enabled & & medium \\
\hline
\end{tabular}

\section{Analysis/Discussion}

Through the conversations a number of links between the environment and the person with schizophrenia were identified. Some involved new ways of understanding known relationships, some refined what was already known, while others challenged our original assumptions. These three approaches to the findings are discussed below together with examples from the conversations. 


\subsection{Author Assumptions That Were Challenged}

Three assumptions were challenged in an obvious way. They were the availability and use of green spaces connected to the home, aspects of information and communication technology and control of thermal conditions:

1. Based on research to date [17-22], there is an understanding that green spaces such as indoor plants, gardens and/or surrounding vegetation are important as restorative agents and support a person's well-being. Thus the authors assumed that residents would reflect favourably on outdoor or garden spaces.

However, it was revealed that although both green (vegetation) and blue (water) spaces were spoken about positively by the majority of participants, for some with schizophrenia, gardens cause additional stress. Variables include the size of the plot or block; the availability of gardening tools, as well as the ability to use them and the cost of purchasing them; and the level of motivation to engage in gardening or going outdoors. For example, Participants \#05 and \#09 found their outdoor spaces overwhelming.

\#05: "the garden's very hard to maintain-a lot of grass-I don't have the tools to do the lawns.No I don't _ ... don't even go out there".

\#09: "Too much gardening is overwhelming. Too much to do. I've just let it go. Grow out of control. ... . I don't water it, I don't prune it, I don't do anything".

In response to a question about spending time outdoors Participant \#08 stated he preferred to be indoors rather than outside in his courtyard.

\#08: "But I don't mind being out there occasionally. If someone comes round and they want a cigarette, I'll go out the back with them".

Implications:

The designer of the garden or other green spaces needs to consider how they will be perceived in terms of forms of engagement, manageability, access and ease of use, as well as the potential running cost. Gardens need to enable alternative modes of interaction without the need for constant tending to plants by the occupant, so the garden retains its beneficial qualities.

2. In Australian society, information and communication technology plays a major role in assisting day-to-day living. As the cost of design and construction is significant, the up-front inclusion of systems and infrastructure that can serve a resident over time, as well as when occupancy changes, is important. Smart technology is increasingly being developed and applied to accommodation for people with disabilities with the aim of improving their QoL. It had been assumed that these developments would assist residents to live comparable lives to the general population, as well as providing support in aspects that may be more difficult for those with schizophrenia.

However, during the conversations a more nuanced understanding arose. Of those interviewed, some people do not have a mobile phone, or a limited or no ability to receive incoming calls. Others do not have a computer or may have controlled access to some services. These limitations are related to an individual's cognitive abilities and financial situation. For example, [23] reports that people with schizophrenia may be unable to engage openly with such technology and are often excluded.

This raises issues of inclusion and exclusion within a technologically literate and dependent society. All participants had access to basic household items such as a televisioneven if the usage varies such as reducing or controlling hours of viewing because 'it drives me nuts having it on all the time' (\#07) and 'I try to get away from television now-not watch as much' (\#09). In addition, technology has allowed some occupants to access books through an e-library via an iPad (\#07) for learning and entertainment, or to satisfy their interest in collecting records, compact discs and books via eBay (\#08 and \#11), while two others (\#02 and \#06) do not use a computer at all.

Due to the current COVID-19 epidemic, an elevated rate of online shopping is normal in mainstream society. This can be particularly advantageous for those with schizophrenia, 
as it allows them to avoid the pressure of being in shops or visiting shopping centres which can lead to anxiety or even to being so overwhelmed that they need to withdraw. For example, participants \#05 and \#02 related their experiences:

\#05: "But I don't like going to the shops. ... Lots of people and crowds of people, I don't like it. [makes me feel] anxious ... That's part of that illness".

\#02: "like when I go to shops ... I still get attacks and things like that at the shops ... I get anxious. And sometimes when I used to go to the shops before, when I was really bad, I'd sometimes say I have to go home NOW".

However, online shopping requires that appropriate infrastructure be available as well as knowledge of and access to digital devices.

R: "So can you get stuff delivered?".

\#05: "You have to be online-to buy online-you need a computer-I'm not on the internet so I'm limited in what I can do".

Implications:

Although state of the art technology and associated connections must be provided at the time of building and renovating, other considerations are also important. ADL must be able to be supported in alternative ways, and/or engagement with technology will need to be developed to be more user-friendly for people with ICF such as those with schizophrenia.

3. It is also well researched that the temperature of the environment can impact a person's sense of wellbeing and their mental health, so it could be assumed that a means to readily adjust thermal comfort via an air-conditioner is a critical consideration. Even so, air conditioning is not a standard fixture in public housing in Perth. The acquisition of an air-conditioner is the responsibility of the occupant. However, the conversations revealed that a number of variables influence the likelihood of an air-conditioned being installed. These include the cost of purchasing an air-conditioner and running expenses, as well as the available positions to install an air-conditioner so that airflow efficiency to all internal spaces is maximized. Experiences include:

\#07: "Ï prefer ceiling fans. I had the option of having an air-conditioner. My mum paid for it at the end of last year-but I opted for the ceiling fans. I prefer the fresh air. Also - the running cost and the maintenance cost because it would be up to me to maintain it and not Community Housing and I also was worried about the replacement costs because I thought well, if it breaks down, do I have to replace it once it's been installed".

And

\#03: "Ït gets very hot when it's humid and the air-con is in a pretty dicky spot-it's not in the middle - So you have to have the air-con on and a fan here to push the air through the house. Our rooms down there get really, really hot".

And

\#08: "You know I didn't have any aircon-so it was very hot in summer. And it was cold in winter".

In addition, flexible cross ventilation should always be considered. Examples were discussed where cross ventilation was compromised and simple interventions (such as orientation and opening positions or security screens to doors or windows) were noted as advantageous.

The heating of the home is also important but was not given much emphasis during the conversations. That said, the cost of heating spaces and the impact of the interior layout and size on a participant's ability to adequately heat the home were mentioned.

\#04: "so if you didn't have the coverings for the bed you wouldn't be able to afford the electricity anyway-so we're lucky we've got the coverings for the bed. I do have reverse cycle air-con but I don't actually use it for winter". 
And

\#05: "I'd have ... heating".

R: "So if you wanted to get heating you'd have to buy it-the Department wouldn't do it?".

\#05: "No, only if I was older". [meaning that the occupant needs to be classified as a senior (over 65 years of age)].

Implications:

Being too hot or too cold is known to have implications for levels of irritability and anxiety. Overall, reverse cycle air-conditioning in low-cost accommodation should be considered a standard requirement (not just for seniors), together with some form of subsidy for running the air-conditioner. It is necessary to give all residents ongoing choice in temperature control depending on their condition and the climatic context in which they live. Passive strategies should also be incorporated to facilitate lowering running expenses. One participant (\#09) mentioned a desire to have solar panels to reduce energy costs. Without appropriate levels of thermal comfort the resident's wellness is at risk.

\subsection{Known Aspects Developed}

Two known aspects of RAD were able to be developed. They related to the location of the accommodation and the safety and security of the accommodation.

1. The location of the accommodation is known to be important for supporting ADL such as shopping, travel and access to services including doctors and hospitals. Most of the participants mentioned the importance of proximity to a variety of services as well as proximity to places of entertainment or more leisure-related facilities. Participants noted facilities such as the pool (\#01), cinema (\#03), cafes (\#04,\#07) and beach (\#01,\#04,\#09) added to their well-being. In addition, two participants $(\# 05, \# 08)$ mentioned the importance of being located in a small compound where their homes were totally independent and yet they were able to connect with a limited number of neighbours when they chose.

Inappropriate social contexts were also mentioned. Participant \#09 referred to distress created by people using nearby walkways after being at the pub whilst \#11 talked about being dissatisfied living in a suburb that had 'a bad reputation,' where home invasion was an expectation. His home was broken into and he retains a fear of it being broken into again.

Implication:

Home location is one of the core attributes of successful accommodation. It is important to consider proximity to places that provide physical, social and spiritual support as well as more pragmatic needs for ADL, including health care. Furthermore, a social context that negatively impacts a resident should be avoided.

2. Safety and security are another core aspect of accommodation design. During the conversations, these were discussed in a wide variety of ways. In broad terms a sense of safety for residents could be influenced by the behaviour of others (such as theft or abusive language).

However, for people with schizophrenia, the issue of perceived safety can be complex. People with schizophrenia may experience paranoia if they feel they are being observed by people, other animate creatures or even inanimate things.

R: "So having things like security cameras-you didn't like the fact they were looking at you-is that what you mean?"

\#03: "Yeah—cause my head always tells me I am being watched and they put up security cameras. I didn't like it".

The position within the building and the location of security doors is important. For example, participant \#07 was delighted living on an upper floor as she 'doesn't feel vulnerable at all' and the front door at ground level has a keypad and intercom. On the other hand, \#09 
stated that for the well-being of people with schizophrenia they should not live in high-rise buildings as 'it's too easy just to jump off".

The perception of a building's appearance was noted to influence the sense of security for two occupants (\#02 and \#09). When a home was poorly constructed or badly maintained, participants felt unsafe.

\#02: "Not everything has to be new and perfect-but it has to be safe and solid".

Occupant \#05 was adamant about the advantages of having a secure home, but her preference was that it be located within some sort of compound with other homes; thus, security and interaction with other people could both be provided.

Controlling the ability to lock windows (\#09) and see in and out of windows, as well as having flexible window treatments to control both access to sunlight and potential visual intrusion (\#04), were identified as being important to one's sense of control, safety and security. People with schizophrenia often feel as though they are being watched.

\#04: "I like the windows. You can block it out if you want to-you've got the thin curtains that give you a bit of privacy - have that down-you can have it open to look at the big windows-you can do whatever you want with it".

Within their home all participants noted places to which they went when unwell or having an episode. Having a particular safe space is a necessity for people with schizophrenia.

\#02: "Yeah just to be home. I need a quiet place, where no one judges you. Where you can just be by yourself. Yeah the safety in that one room where you can go to all the time".

Implications:

A sense of safety and security is of key importance to residents with schizophrenia. This impacts the choice of site, the design and positioning of security and locking systems and where the unit or home is located vertically in complexes or developments, as well as providing opportunities to create secure spaces within the home and to control physical access and screen views into and out of the residence. All aspects should be considered so that the resident feels safe, secure and can control their privacy both when feeling well and when experiencing episodes.

\subsection{New Aspects Arising}

Three aspects of design that had not been previously identified were raised. These included the sensitivity to perceived lack of order and tidiness, the impact of costs on decision-making and the potential reduced sense of smell.

1. An unexpected finding was the heightened awareness of mess and of dust or dirt in the home. It was evident that residents' sensitivity increased when feeling unwell or having an episode. Therefore, the ability to clean surfaces, to store things in appropriate locations for larger items and to have adequate drawers, cupboards and bench space to undertake daily activities is required.

The design of storage systems needs to be reconsidered so that they are readily adaptable to assist with accessibility and the ease of use of items stored in each of the functional zones. In this study laundry benches, vanity benches, cooking surfaces, drawers and wardrobes were noted as inadequate by some of those interviewed. In addition, however, items need to be able to be out of sight when required and this action carried out with ease and readily understood. It should be noted that the ability to carry out a task may be compromised, thereby challenging typical storage solutions. For example, participant \#02, when talking about all the jars, cans and bottles on the benches and table in the kitchen said:

\#02: "But when I am unwell I want to have it put away but I can't physically do it. But I need it out. My thoughts are not rational. They're rational but not how I would normally think."

R: "There's nothing wrong with the cupboards—so you could put them away if you wanted to?" 
\#02: "Yes, I could put them away. Yeah I could put them away, but umm but with my thoughts, I couldn't do it-because with my thoughts. I'm not well. I couldn't physically do it, because I wouldn't know where I was putting everything. I'd be just shoving it away anywhere and making a bigger mess. But I could do it when I'm well but I don't want to-cause that's how I like to have it".

Although the residence being visited appeared to be very clean and tidy to the researchers, the resident may have perceived that it was not. For example participant \#05 noted the following.

R: "Is there any area of you house - that could be the garden or any of the rooms-that you feel things are the most challenging for you in terms of you being able to cope and live ok?"

\#05: "Every room — the clutter and the mess ... To keep it clean and to be organized-I find it hard".

R: "When you're not feeling well ... when you're feeling very anxious, -are there things that you notice about your house that are, —that impact upon you?"

\#05: "Yeah, I don't like clutter".

R: "So you notice the clutter when you're not feeling well?"

\#05: "Yeah".

Implications:

Storage and display areas need to be designed to cater for those with a heightened awareness of clutter, mess and cleanliness. Traditional solutions may need to be rethought to cater seamlessly for periods of wellness and reduced wellness.

2. A second aspect that was not initially understood was the influence that the actual or perceived running costs have on choices and behaviours, and in turn their impact on lifestyle. Issues raised by participants ranged from a lack of a place to wash the car, the lack of means for heating or cooling, maintenance costs, the requirement for tools and equipment to establish and maintain a yard or garden and the cost to maintain a pet even though its value was recognised.

\#05: "Yeah I'd like a pet-but It costs money, time and effort".

\#03: "We've got 2 parking spots out there at the moment-but you're not allowed to wash your car out there or anything-you can't really work on your car out there-it's in the strata rule-so if you want to wash your car you have to take it to one of the places and pay to get it to be washed".

Implications:

Not being able to afford to purchase or to maintain aspects of the home can have direct impact for a person's well-being. Therefore when designing residences, consideration needs to be given to the provision of essential items, such as air-conditioners, and facilities that allow for easy use and for maintenance to occur. For example, garden areas should allow for resident input but be pleasant places if a particular resident cannot contribute to the upkeep of external areas due to lack of funds. This may assist in reducing a sense of helplessness, being overwhelmed or adding to a lack of motivation.

3. People with schizophrenia perceptually connect to more/different sensory elements than people without the cognitive impediment. Sensory pollution from noise, glare or heat/cold are acknowledged as possible triggers for hallucination. However, people with schizophrenia may not have olfactory sensibilities [24,25]. One participant (\#07) referred to her lack of sense of smell when discussing the position of the kitchen exhaust fan, which was not directly above the hot plate.

\#07: “Öh, well I have no sense of smell anyway. I think I remember years and years ago when I was a little kid smelling that lavender water in my grandma's room. But haven't got a lot of sort of memories of smell". 
Implications:

Absence of a sense of smell means that an occupant may not be aware of smells emanating either from their own home or from other spaces in a building they occupy such as foyer or lobby spaces. These smells may lead to others judging the person's worth and position in society. Further, this raises the necessity of having well-maintained smoke alarms as an occupant may not respond to the smell of smoke in the early stages of a fire.

\subsection{Insights into Experience of Schizophrenia Provided by Those Interviewed}

The participants spoke about situations that had made them personally feel anxious or depressed (for example). However, there were three aspects highlighted that participants had observed in people with schizophrenia more broadly. These were a tendency to collect or to hoard items, an ongoing concern about housing tenure and a need to be engaged in some activity to assist their condition:

1. It was noted that people with schizophrenia are likely to collect items and the researchers observed that some participants had collections of books, CDs and records (\#01, $\# 06, \# 08, \# 11)$, whilst others had excess items relative to the space available (\#02, \#11).

\#08: "Ït seems to be common with people with my kind of condition. So what I'm saying is that I think it's essential that they have some sort of storage of some sort.-For all their stuff you know. I've just noticed that."

Participants \#06 and \#08 raised the need for more storage to accommodate their hobbies. Participant \#06 had been able to fit-out an outdoor storeroom with shelving to store his artwork, but Participant \#08, who collected books, CDs and records, found himself gradually being squeezed into less and less space for ADL.

He explained:

\#08: “Ï look around and everything's sort of looks ok-structured badly ... I've got to do something about it-even if I don't get rid of some of these things-which would be really upsetting - it's taken so long to get it together - or I have to find an alternate solution."

R: "So can I just clarify, when you're not feeling so well it's actually because it feels cluttered -is that what you're saying?"

\#08: "No, it's knowing that other people know that it's cluttered-you know what I mean-But because other people brought that to my attention I'm aware of that. The clutter. When I visited other people's places, I've noticed how much they collect stuff as well. It seems to be common with people with my kind of condition".

Implications:

The authors of [26] have indicated that some people with schizophrenia have a tendency to hoard or to collect items and in fact may perceive it as an aspect of their identity. Some people with schizophrenia derive personal satisfaction from collecting, so some accommodation for this could be provided. This trait may indicate that storage areas or rooms need to be considered more carefully.

2. A number of participants raised the issue of housing tenure and noted that a state of uncertainty negatively impacted their wellbeing and need for care. At the time of the interviews, the housing tenure of participants varied from private ownership by the occupant (two participants), private ownership by parents of the occupant, (three participants) joint ownership between occupant and a public housing authority (two participants) and public housing rental (four participants). However nearly all participants had, at some point, lived in temporary housing supplied by a service provider and made comments about that experience.

\#10: "Then I went to a different unit and so on and so on and during that time, there were times when I ended up in hospital, - and a lot of that had to do with the fact that I didn't have that continuity of, umh, housing".

Participant \#10 also stated that people with schizophrenia who are on a disability pension commonly believe that home ownership is impossible for them, even though "the 
one thing about people with schizophrenia is that ... can't handle change". He had managed to become a homeowner and believed "the most steadying influence I've ever had in my life is owning this place." In a similar vein participant \#08 said "just having this place has made my life a lot better... my own environment. I own it ..."."

\#07: "Ï've gone from one service to the next, because none of them provide permanent ahh permanency. No permanent accommodation. You're only there for 4 years-so you've always got that anxious feeling and you can never really call a place home. You know".

In addition, it was also noted how those who rent low-cost housing seem to be reluctant to report damage or problems in their accommodation, perhaps fearing they may be asked to leave the property. Participants \#06, \#07 and \#08 all provided examples of this.

Participant \#06 reported that he had rented the same home for 22 years and the house was inspected every year.

\#06: "Ï always pass though because I keep the place so lovely-spotless and clean".

However it was obvious to the researchers that the veneer on the kitchen cupboards was badly delaminating and broken off in places and was clearly in need of replacement.

Participant \#07 spoke about the wardrobe in her bedroom having no doors although clearly the wardrobe was designed to have doors. The doors had been removed by a previous tenant and the participant was saving up to buy wardrobe doors rather than approaching the lessor to have doors reinstalled on the wardrobe.

Participant \#08 recounted his inexplicable reluctance to have a new stove and washing machine installed by a new landlord in the flat he was renting.

\#08: "The stove-they actually asked me if I wanted a new stove-or a washing machine, because there were taps in the [bathroom] for a washing machine. And for some reason I turned them down for both of those things. I could have done with those-that would have made life a little bit easier".

Implications:

Research to determine the social and economic costs of care versus the cost of providing secure housing options for this tenant group is required to inform efficient and effective tenancy models. In addition, public housing agencies could be more proactive in regularly providing financial advice to tenants to see if / how homeownership can become a reality for more people with schizophrenia.

The approach to the maintenance of rental accommodation for people with schizophrenia needs to be more carefully considered. Maintenance inspections may need to be more proactive and/or more robust interior finishes with a longer life should be considered.

3. A number of participants commented on how a lack of being engaged with an activity can impact a person's mental state. For example, \#03 stated:

“. . . because I find if I keep busy I'm not paying too much attention to my voices. So I do a lot of group work just to keep myself busy during the day".

Having space within the home to engage in hobbies or to be able to access interests nearby also serves this purpose. For example, \#01 spoke of the benefits of swimming in the local pool which he found 'invigorating,' while \#02 stated:

"... sometimes when you don't want to go outside because you are self-conscious about walking. If you've got a treadmill-in a room. Exercise is important-It makes a difference when I'm doing exercise to music".

Participant \#04 noted the advantages of going for a drive to the beach or the shops.

\#04: "When I'm unwell I need to get out of the house because I spend too much time in the house-I get too ... caught up in the housework."

Implications:

Location selection can facilitate people getting out of their homes and avoiding obsessing or being immersed in their own thought, which can be detrimental. Internally, 
providing sufficient space or flexibility in how spaces can be used could cater to resident interests.

\subsection{Summary}

To summarize, the findings provided insights into what had been identified previously through the literature and prior work. Conversations with the people that we interviewed gave nuance to what was known. There were few new aspects; however, some challenged the researchers' assumptions, while others were seen in a new light. The examples provided above have direct implications for those designing accommodation for people with schizophrenia who are living independently in houses and units.

\section{Conclusions}

The current study provides insights into how a condition, which impacts cognitive functioning, influences the experience of living in a house or unit. The impacts include aspects relating to how residents understand, manage and experience their environment, as well as its potential meaning.

The study highlights the advantages of including the potential resident-group's experiences. As researchers and designers we were challenged to test our assumptions and are appreciative of having our understanding developed. Besides gaining insights into the lives of people with schizophrenia, there are new concepts that arose in the study which had not arisen previously - at least not with the nuances introduced by those we interviewed. Therefore, this approach is recommended for informing design practice. This more nuanced understanding will enable design propositions to be developed more purposefully to better serve a resident with schizophrenia.

The current study lays the foundations for a user-focused approach to design. With an increasing number of people experiencing compromised mental health, it also aims to open up the debate to ensure that the built environment can support the well-being and QoL of all users and to recognize that new and evolving approaches may be needed to achieve this end.

\section{Future Research}

For each of the categories described in the findings, more in-depth study is required. That is, the study of the implications for both environmental design and the impact on the residents of:

- the tendency to collect or to hoard items,

- the reduced sense of smell,

- the ongoing concern about housing tenure,

- the need to be engaged in some activity to assist their condition,

- the sensitivity to a perceived lack of order and tidiness,

- the impact of costs on decision-making,

- the location of accommodation and safety,

- the perceived security of accommodation,

- the availability and use of green spaces connected to the home,

- information and communication technologies access and useability, and

- the control of thermal conditions in the home.

Although it complies with the requirements for qualitative conversational interviews, the research would be enriched by interviewing more participants with experiences drawn from a more diverse range of living situations and across various demographic groups.

In the future, it is also planned to use the RAD-ICF approach to design residences for people with ICF and to interrogate these design resolutions, as well as to interview the occupants living in those homes as part of an evaluative strategy. 
Author Contributions: Both authors participated in the conceptualization, methodology, data collection, formal analysis, resources and all stages of writing. Both authors have read and agreed to the published version of the manuscript.

Funding: This research was partly funded by Alastair Swayn Foundation, Grant ID ASFR006.

Institutional Review Board Statement: The study was conducted according to the guidelines of the Declaration of Helsinki, and approved by the Human Research Ethics Committee of CURTIN UNIVERSITY (HRE 2020-0391) on 17 July 2020.

Informed Consent Statement: Informed consent was obtained from all subjects involved in the study.

Data Availability Statement: Restrictions apply to the availability of these data. Data obtained from the participants is available from the authors with the permission of the participants.

Acknowledgments: The support of Neami National to identify some participants willing to be part of this research was invaluable.

Conflicts of Interest: The authors declare no conflict of interest.

\section{References}

1. Karol, E.; Smith, D. Expanding design approaches to create supportive home environments for people with impeded cognitive functioning. Des. Health 2020, 4, 43-62. [CrossRef]

2. Smith, D.; Karol, E. An accommodation design matrix to assist the provision of supportive accommodation for people with altered cognitive functioning. Des. Health 2019, 3, 261-282. [CrossRef]

3. Karol, E.; Smith, D. Impact of design on emotional, psychological or social well-being for people with cognitive impairment. HERD J. 2018, 12, 220-232. [CrossRef] [PubMed]

4. NSW Law Reform Commission. People with Cognitive and Mental Health Impairments in the Criminal Justice System; Report 135; NSW Law Reform Commission: Sydney, Australia, 2012; ISBN 978-0-7447-2667-4.

5. Summer Foundation. Living Like Everyone Else: Re-Thinking 24-Hour Support Specialist Disability Accommodation (SDA) Payments; A Guide for NDIS Participants \& Providers; Summer Foundation: Blackburn, Australia, 2017; Available online: https://www. summerfoundation.org.au/wp-content/uploads/2017/03/living-like-everyone-else-for-web.pdf (accessed on 5 February 2021).

6. Independent Living Villages. Housing after Hospital. Supported, Specialist Independent Living. Who is SDA for? Available online: https:/ / ilv.org.au/sda-housing-after-hospital/ (accessed on 16 February 2021).

7. Boys, J. Doing Disability Differently. The Architectural Review. 6 September 2014. Available online: https://www.architecturalreview.com/essays/doing-disability-differently (accessed on 9 March 2021).

8. Arya, A.; Sindhwani, G.; Kadian, R. Neurotransmitter and brain parts involved in schizophrenia. Asian J. Pharm. Clin. Res. 2018, 11, 4-11. [CrossRef]

9. Goines, L.; Hagler, L. Noise Pollution: A modern plague. South. Med. J. 2007, 100, 287-294. [CrossRef] [PubMed]

10. Wright, B.; Peters, E.; Ettinger, U.; Kuipers, E.; Kumari, V. Effects of environmental noise on cognitive (dys)functions in schizophrenia: A pilot within-subjects experimental study. Schizophr. Res. 2016, 173, 101-108. [CrossRef] [PubMed]

11. Carlsson, I.; Blomqvist, M.; Jormfeldt, J. Ethical and methodological issues in qualitative studies involving people with severe and persistent mental illness such as schizophrenia and other psychotic conditions: A critical review. Int. J. Qual. Stud. Health Well Being 2017, 12 (Suppl. S2), 1368323. [CrossRef] [PubMed]

12. Guest, G.; Bunce, A.; Johnson, L. How Many Interviews Are Enough? An Experiment with Data Saturation and Variability. Field Methods 2006, 18, 59-82. [CrossRef]

13. Hennink, M.M.; Kaiser, B.N.; Marconi, V.C. Çode Saturation Versus Meaning Saturation: How Many Interviews Are Enough? Qual. Health Res. 2017, 27, 591-608. [CrossRef] [PubMed]

14. Strauss, A. Qualitative Analysis for Social Scientists; Cambridge University Press: Cambridge, UK, 1987.

15. Strauss, A.; Corbin, J. Grounded theory methodology: An overview. In Handbook of Qualitative Research; Denzin, N., Lincoln, Y., Eds.; Sage Publications: Thousand Oaks, CA, USA, 1994; pp. 273-285.

16. Glaser, B.; Holton, J. Staying open: The use of theoretical codes in grounded theory. Grounded Theory Rev. 2005, 5, 1-20.

17. Ulrich, R.S. View through a window may influence recovery from surgery. Science 1984, 224, 420-421. [CrossRef] [PubMed]

18. Ulrich, R.S. Effects of interior design on wellness: Theory and recent scientific research. J. Health Int. Des. 1991, 3, 97-109.

19. Ulrich, R.S.; Zimring, C.; Zhu, X.; DuBose, J.; Seo, H.; Choi, Y.; Quan, X.; Joseph, A. A review of the research literature on evidence-based healthcare design. HERD J. 2008, 1, 61-125. [CrossRef] [PubMed]

20. Kaplan, K. The restorative benefits of nature: Toward an integrative framework. J. Environ. Psychol. 1995, 15, 169-182. [CrossRef]

21. Kaplan, R. The nature of the view from home: Psychological benefits. Environ. Behav. 2001, 33, 507-542. [CrossRef]

22. Connellan, K.; Gaardboe, M.; Riggs, D. Stressed spaces: Mental health and architecture. HERD J. 2013, 6, 127-168. [CrossRef] [PubMed]

23. Wong, K.; King, D.; Balzan, R.; Liu, D.; Galletly, C. Technology literacy and engagement in people with schizophrenia. Schizophr. Bull. 2018, 44 (Suppl. S1), S208. [CrossRef] 
24. Chen, X.; Xu, J.; Li, B.; Guo, W.; Zhang, J.; Hu, J. Zfactory impairment in first-episode schizophrenia: A case-control study, and sex dimorphism in the relationship between olfactory impairment and psychotic symptoms. BMC Psychiatry 2018, 18, 199. [CrossRef] [PubMed]

25. Moberg, P.J.; Agrin, R.; Gur, R.E.; Gur, R.C.; Turetsky, B.I.; Doty, R.L. Olfactory Dysfunction in Schizophrenia: A Qualitative and Quantitative Review. Neuropsychopharmacology 1999, 21, 3. [CrossRef]

26. Schoua, M.; Urfer-Parnas, A.; Parnas, J. The hoarding phenomenon in schizophrenia spectrum disorders. Psychopathology 2020, 53, 111-117. [CrossRef] [PubMed] 